\title{
Far-infrared therapy for secondary vascular access patency of hemodialysis patients
}

\author{
Hiroaki $\mathrm{l}^{1^{*}}$, Junichiro Nakata ${ }^{2}$, Rumi Aoyama ${ }^{2}$, Hiroyuki Inoshita' ${ }^{1}$ Takanori Nakano ${ }^{1}$, Masanori Ishizaka', \\ Mitsumine Fukui ${ }^{3}$, Yasuhiko Tomino ${ }^{3}$ and Yusuke Suzuki ${ }^{2}$
}

\begin{abstract}
Introduction: Far-infrared radiation therapy (FIRT), which utilizes an invisible electromagnetic wave, has demonstrated its ability to improve vascular access flow for hemodialysis (HD) patients. Although previous overseas studies revealed the beneficial effects of FIRT on HD patients by improving vascular function, no study in Japan has examined the clinical efficacy of FIRT for such patients. Therefore, we aimed to evaluate the usefulness of FIRT for HD patients. We paid particular attention on secondary vascular access (VA) patency of HD patients.

Methods: Eighteen patients who had undergone vasodilation of VA vessels or vascularization more than once were selected from approximately 70 outpatients receiving HD in the Dialysis Unit of the Juntendo University Hospital. FIRT was administered for 40 min during HD three times per week for 1 year to the not frequent VA treatment (nf-VAT) group, for which VA intervention had been performed no more than two times during the 1 year, and to the frequent VA treatment (f-VAT) group, for which VA intervention had been performed three times or more over the 1 year. Variables including VA treatment interval were compared between those groups.

Results: The nf-VAT group did not require VA re-treatment during the study period. The VA treatment interval was significantly prolonged in the f-VAT group. The fistula diameter expanded, and intimal thickening improved significantly in the nf-VAT group. Oxidized low-density lipoprotein cholesterol levels decreased slightly.

Conclusion: This study suggested that FIRT is effective for improving secondary VA patency.

Trial registration: UMIN, UMIN000011965. Registered 4 October 2013, Japanese: https://upload.umin.ac.jp/cgiopen-bin/ctr/ctr_view.cgi?recptno=R000013960, English: https://upload.umin.ac.jp/cgi-open-bin/ctr_e/ctr_view. cgi?recptno=R000013960
\end{abstract}

Keywords: Far-infrared therapy, Hemodialysis, AVF stenosis, Intervention AVF, Oxidative LDL

\section{Introduction}

Vascular access (VA) is necessary for patients on maintenance hemodialysis (HD). The prognosis of VA in HD patients is presumably influenced by the following factors: enhanced coagulation properties, injury to vascular endothelial cells, red blood cell volume, and gene polymorphisms in heme oxygenase-1 (HO-1) gene [1]. Since these factors can be controlled by drugs or other therapies, they have been of interest to researchers in this field. Approximately 80 to $85 \%$ of VA failures in HD patients

\footnotetext{
* Correspondence: hiroaki@juntendo.ac.jp

1Department of Nephrology, Juntendo University Nerima Hospital, 3-1-10

Takanodai Nerima-ku, Tokyo 177-0033, Japan

Full list of author information is available at the end of the article
}

result from thromboses, and more than $80 \%$ of these are caused by underlying stenosis [2].

Infrared radiation utilizes an invisible electromagnetic wave with a wavelength that is longer than that of the visible light. Previous studies about far-infrared radiation therapy (FIRT) have demonstrated its ability to improve skin blood flow. Indeed, FIRT has been used for the treatment of ischemic lesions or skin necrosis due to injury, diabetes, or peripheral nerve disease. Such studies have suggested that FIRT may improve vascular endothelial function and decrease endothelial dysfunction in patients with coronary artery disease, heart failure, and arrhythmia [3, 4]. One previous study in Taiwan examined the effects of FIRT in HD patients and showed that it steadily increased arteriovenous fistula (AVF) blood

(c) The Author(s). 2019 Open Access This article is distributed under the terms of the Creative Commons Attribution 4.0 International License (http://creativecommons.org/licenses/by/4.0/), which permits unrestricted use, distribution, and reproduction in any medium, provided you give appropriate credit to the original author(s) and the source, provide a link to the Creative Commons license, and indicate if changes were made. The Creative Commons Public Domain Dedication waiver (http://creativecommons.org/publicdomain/zero/1.0/) applies to the data made available in this article, unless otherwise stated. 
flow. Compared with the control group, the treatment group, who received FIRT for 1 year, demonstrated a lower rate of AVF failure (12.5\% vs 30.1\%), increased AVF blood flow, and a higher primary patency rate (85.9\% vs $67.6 \%$ ) [5]. In Japan, however, no study has examined the clinical efficacy of FIRT. In addition, no study in Japan has examined the effects of FIRT with regard to secondary VA patency.

Primary AVF patency rate at 1 year after HD introduction in the Juntendo University Hospital is $64 \%$, which is similar to the mean AVF patency rate reported by the Japanese Association of Dialysis Physicians. This study examined the efficacy of FIRT for secondary VA patency in HD outpatients at our hospital.

\section{Methods}

The instrument used in this study was the WS FarInfrared Therapy Unit (WS Far IR Medical Technology, Taipei, Taiwan). A total of 18 patients who had undergone vasodilation of VA vessels or revascularization (standard radial artery to cephalic vein fistula) more than once were selected from approximately 70 outpatients receiving HD at the Dialysis Unit in the Juntendo University Hospital. FIRT was irradiated for $40 \mathrm{~min}$ during HD three times per week with the radiator placed 20 to $30 \mathrm{~cm}$ from the skin of the limb with the fistula. Comparisons were made between 13 patients who had undergone percutaneous trans-angioplasty (PTA) due to fistula (or in the vicinity) stenosis two times or fewer over the past year (not frequent VA treatment [nf-VAT] group) and five patients who had undergone PTA three times or more over the past year (frequent VA treatment [f-VAT] group). Patient characteristics are noted in Table 1.

The intervention criterion by VA treatment is the need for any interventional procedure (surgery or angioplasty) to correct an occlusive or malfunctioning fistula which could not sustain an extracorporeal blood flow during HD after excluding the following stenosis-unrelated events, such as infectious complication, progressive

Table 1 Patient background characteristics

\begin{tabular}{lll}
\hline & f-VAT group & nf-VAT group \\
\hline Number of patients & 5 & 13 \\
Sex (male to female) & $2: 3$ & $8: 5$ \\
Age (years) & $70 \pm 12.5$ & $69.0 \pm 12.8$ \\
$\begin{array}{l}\text { Diabetes patients (\%) } \\
\text { Dialysis vintage (months) }\end{array}$ & 66.7 & 0 \\
$\begin{array}{l}\text { Percentage of patients on } \\
\text { anticoagulants (\%) }\end{array}$ & $91.3 \pm 127.9$ & $113.0 \pm 56.4$ \\
$\begin{array}{l}\text { The number of annual VAT } \\
\text { times before intervention } \\
\text { by FIRT }\end{array}$ & $5.6 \pm 0.9$ & 36 \\
\hline
\end{tabular}

aneurysmal formation, or steal syndrome. The effects of FIRT were evaluated at baseline ( 0 week: the day on PTA was performed) and at 4, 12, 24, 48, and 96 weeks. Blood vessel ultrasonography was performed before dialysis to measure fistula blood flow, intima thickness, and degree of stenosis. The degree of fistula sclerosis was evaluated for the brachial artery (BA) flow and resistance index (RI) measuring with ultrasonography. Blood tests included indices of renal anemia (hemoglobin, hematocrit, serum iron, total iron-binding capacity, ferritin, and red blood cell volume distribution width) and progression of arteriosclerosis (total homocysteine, highly sensitive C-reactive protein, oxidized LDL-C, pentosidine, and VEGF).

This study was approved by the Ethics Committee of the Juntendo University (approval no. 13-085) and registered with the University Hospital Medical Information Network (UMIN000011965). After an explanation of the procedures, oral and written informed consents were obtained from all patients.

The statistical analysis was performed using JMP 10 (SAS Institute, Cary, NC, USA). Standard descriptive statistics was used to assess the baseline characteristics. Data are presented as mean \pm standard deviation. Repeated-measures ANOVA was performed to compare serial changes in the clinical data and echo parameters. $P$ values $<0.05$ were considered statistically significant.

\section{Results}

The nf-VAT group did not require VA re-treatment during the study period. The f-VAT group was treated with PTA which interval for thrice or more over the past year. The number of VA treatment episodes for nf-VAT group was 1.0 per patient-year before FIRT and zero time after FIRT. The number of VA treatment episodes for f-VAT group was 5.6 per patient-year before FIRT

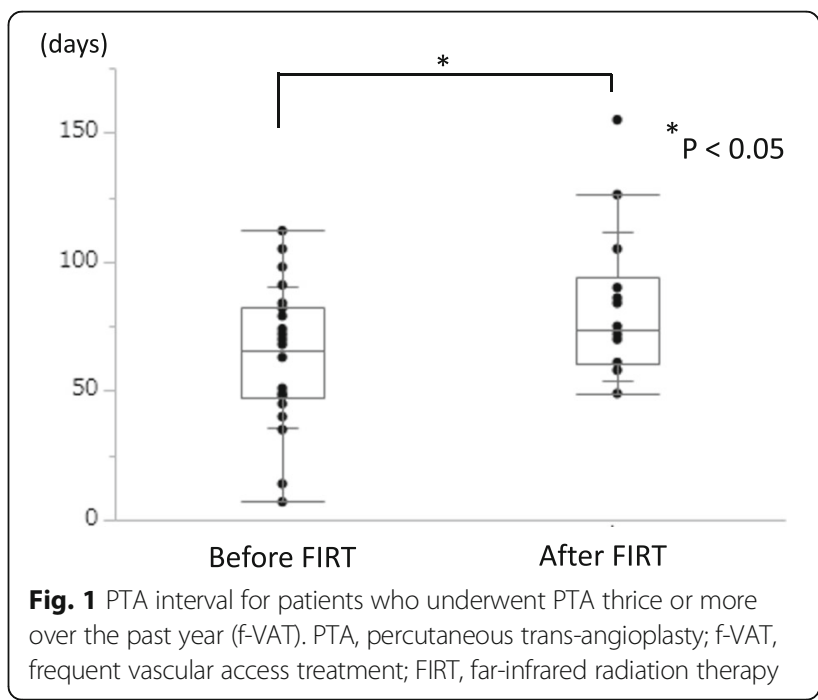



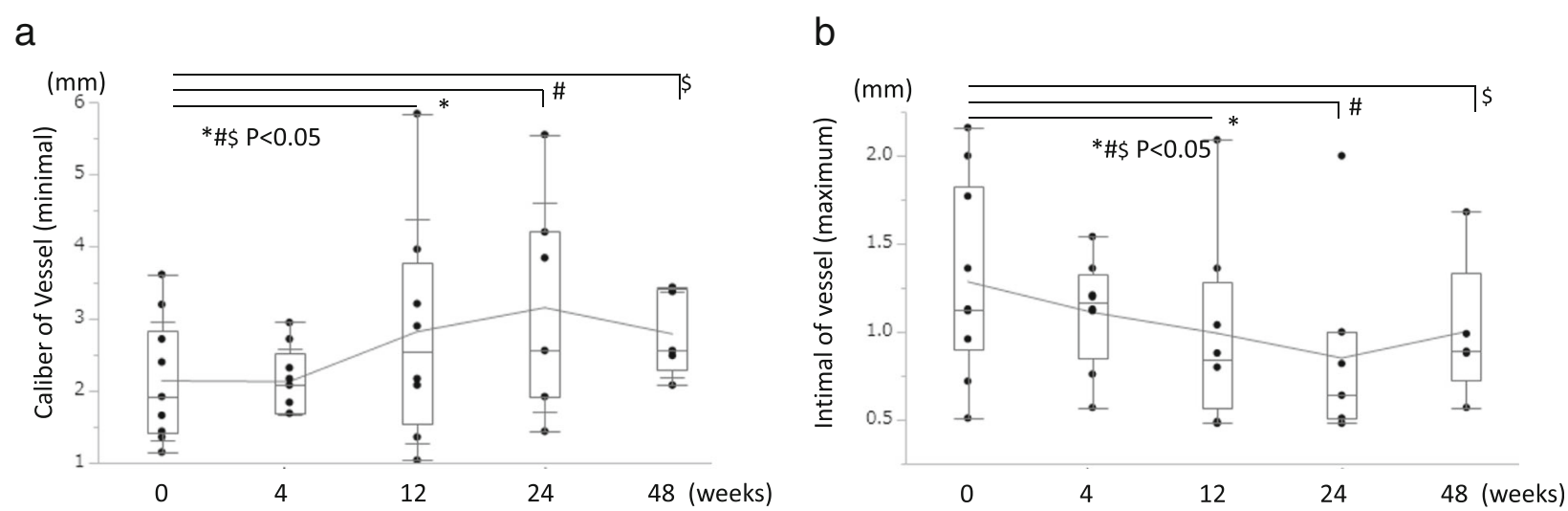

Fig. 2 Fistula diameter (a) and intimal thickening (b) for patients who underwent PTA twice or less over the past year (nf-VAT). nf-VAT, rare vascular access treatment; nf, not frequent; PTA, percutaneous trans-angioplasty

and 4.4 per patient-year after FIRT. The f-VAT group showed in the mean VA treatment interval from $62.9 \pm$ 27.3 days to $83.4 \pm 29.6$ days (Fig. 1 ). The nf-VAT group was evaluated measuring the fistula diameter and intimal thickening for patients who underwent PTA twice or less over the past year. The nf-VAT group showed significantly increase in cephalic vein (fistula) diameter and decreased in intimal thickening (Fig. 2). The f-VAT group showed not significantly increase in cephalic vein (fistula) diameter and not decreased in intimal thickening (Fig. 3). f-VAT group patients were need for VA intervention within 24 weeks. The nf-VAT group was measuring for evaluation of vascular endothelial growth factors. Although there was no significant change in serum VEGF, a significant decrease in oxidized LDL-C level was observed (Fig. 4). Renal anemia (hemoglobin, hematocrit, serum iron, total iron-binding capacity, ferritin, and red blood cell volume distribution width) and progression of arteriosclerosis (total homocysteine, highly sensitive C-reactive protein) were not significantly difference between before and after FIRT (data not shown).

Brachial artery (BA) flow and resistance index (RI) were evaluated after FIRT. BA flow and RI after FIRT were measured at 0,4 , and 12 weeks in the $\mathrm{f}$-VAT group. BA flow and RI after FIRT were measured at 0, 4, 12, 24, 48 , and 96 weeks in the nf-VAT group. In the f-VAT group (done for PTA each about 3 months), fistula blood flow measured in the brachial artery showed a tendency to decrease, whereas the vascular RI showed a tendency to increase (Fig. 5a). In the nf-VAT group, no significant changes in fistula blood flow (tendency to increase) or RI (tendency to decrease) were observed (Fig. 5b).

\section{Discussion}

This study examined the therapeutic effects of FIRT on secondary VA patency in HD patients and suggested that FIRT may suppress vascular endothelial proliferation, thereby improving secondary VA patency. The
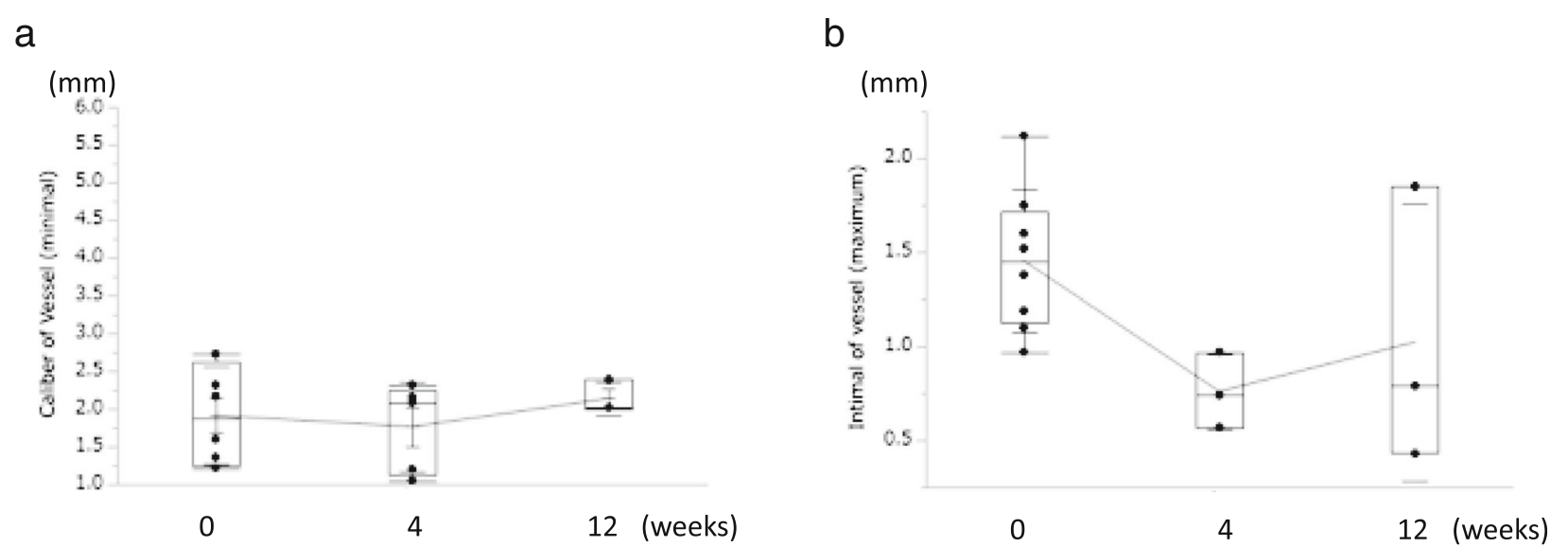

Fig. 3 Fistula diameter (a) and intimal thickening (b) for patients who underwent PTA thrice or more over the past year (f-VAT). PTA, percutaneous angioplasty; f-VAT, frequent vascular access treatment 

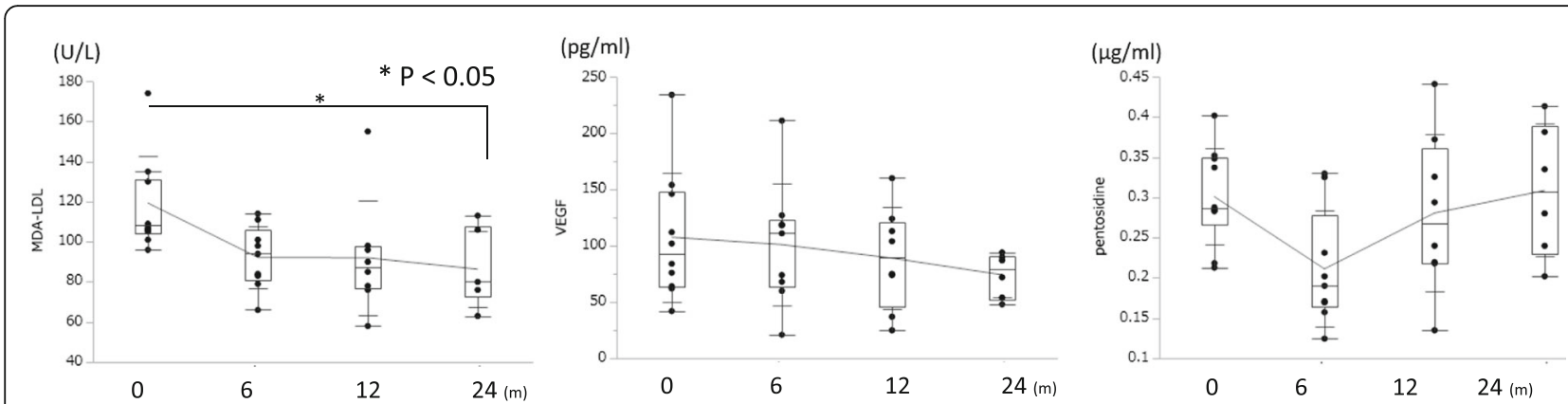

Fig. 4 Evaluation of vascular endothelial growth factor in the not frequent vascular access treatment (nf-VAT) group. MDA-LDL, malondialdehydemodified low-density lipoprotein; VEGF, vascular endothelial growth factor

mechanism of action of FIRT is discussed in the combination of both short-term thermal and long-term nonthermal effects that increase VA blood flow [5]. The short-term thermal effects of FIRT are vasodilation and increased VA blood flow. It has been reported that the temperature can be increased by $4{ }^{\circ} \mathrm{C}$ in tissue with a depth of $10 \mathrm{~mm}$. With radiation administered at a distance of $20 \mathrm{~cm}$ above the skin surface for 30 to 60 min, the skin temperature steadily increased to a plateau of 38 to $39^{\circ} \mathrm{C}$. Regarding the non-thermal effects, animal and clinical studies have suggested the possibility that far-infrared radiation can improve endothelial function. In terms of the effect on oxidative stress, patients receiving $45 \mathrm{~min}$ of FIRT dry sauna daily for 2 weeks had

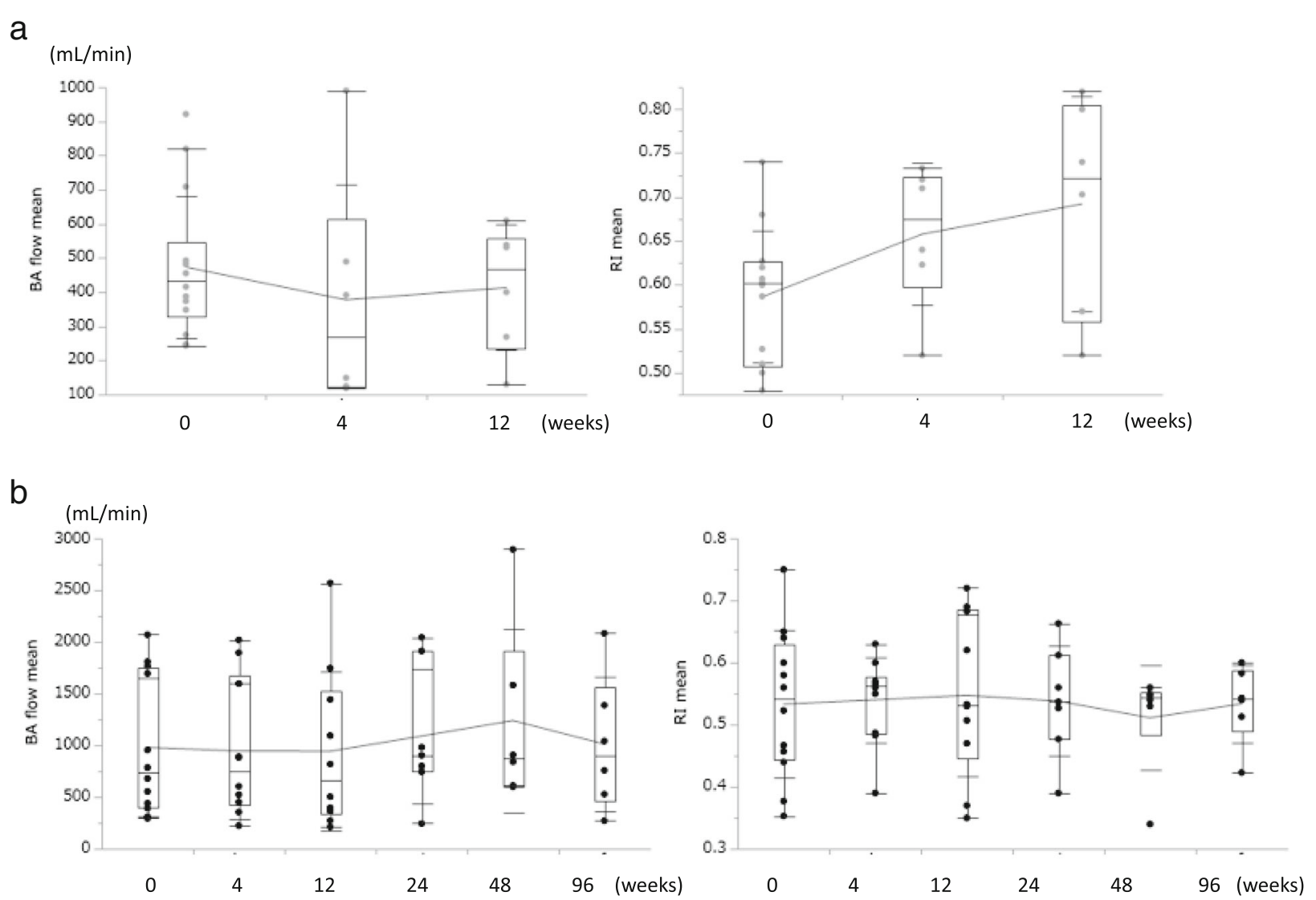

Fig. 5 BA flow and RI before and after FIRT. a BA flow and RI before FIRT and 4, 12, and 24 weeks after FIRT for f-VAT group. b BA flow and RI before FIRT and 4, 12, 24, 48, and 96 weeks after FIRT for the nf-VAT group. BA: brachial artery; RI: resistance index; FIRT: far-infrared radiation therapy; f-VAT: frequent vascular access treatment, nf-VAT: not frequent vascular access treatment 
significantly lower urinary levels of 8-epiprostaglandin F2 $\alpha$ (a marker of oxidative stress in vivo) than the control group [6].

VA patency in HD patients is influenced by many mechanical and medical factors. FIRT is expected to increase VA blood flow and improve primary and secondary AVF patency through its anti-inflammatory effects. Studies report that these mechanisms of FIRT are related to its thermal effects as well as its non-thermal effects that promote proliferation of the vascular endothelium via stimulation of HO-1 [7] and suppression of tumor necrosis factor-induced adhesion molecules [8]. Measurements of the indices of arteriosclerosis, oxidative stress, and vascular endothelial growth factor (VEGF) may contribute to our understanding of the mechanisms of fistula stenosis and thrombus formation as well as the mechanism of FIRT. Previous study reported that oxidative low-density lipoprotein (LDL) is frequently found in the absence of monocyte/macrophages, whereas the opposite is rare, suggesting that intimal LDL accumulation and oxidation contributes to monocyte recruitment in vivo [9]. Furthermore, near-infrared heat lamp therapy for a short time can effectively prevent the lipid disorders induced by radiation through the positive modulation mechanism of paraoxonase 1 (PON1) and myeloperoxidase (MPO) enzymes and improvement of oxidative stress [10]. We believe that FIRT is useful not only for improving AVF patency but also for preventing vascular disease complications. Increasing AVF patency is important for maintaining fistula blood flow, which is necessary to achieve sufficient dialysis efficiency. In addition, FIRT may alleviate psychiatric distress and improve activities of daily living for patients with frequent VA trouble (VA failures), potentially leading to reduced healthcare costs.

The most obvious limitation of this study is the lack of control group and control phase. This study was limited because all previous studies were conducted in Asia. Therefore, evidence regarding the effectiveness of FIRT for enhancing the patency of AVF for people of other ethnicities is lacking.

\section{Conclusion}

Our results suggest that FIRT might suppress proliferation of the vascular endothelium and is useful for improving secondary VA patency.

\footnotetext{
Abbreviations

AVF: Arteriovenous fistula; BA: Brachial artery; FIRT: Far-infrared radiation therapy; f-VAT: Frequent VA treatment; HD: Hemodialysis; HO-1: Heme oxygenase-1; LDL: Low-density lipoprotein; MPO: Myeloperoxidase; nfVAT: Not frequent vascular access treatment; PON-1: Paraoxonase 1; PTA: Percutaneous trans-angioplasty; RI: Resistance index; VA: Vascular access; VEGF: Vascular endothelial growth factor
}

\section{Acknowledgements}

We are indebted to the nephrologists and patients at the Juntendo University Hospital for their collaboration in this study, and many thanks to WS Far IR Medical Technology, Taipei, Taiwan, complimentary used in this study for WS Far-Infrared Therapy Unit.

\section{Disclosures}

None

\section{Authors' contributions}

$\mathrm{HI}$ contributed to the research idea and study design. RA, HI, TN, and MI contributed to the data acquisition. HIo, RA, and MF contributed to the data analysis/interpretation. YT and YS are the research directors. Each author contributed important intellectual content during manuscript drafting or revision and accepts accountability for the overall work by ensuring that questions pertaining to the accuracy or integrity of any portion of the work are appropriately investigated and resolved. All authors read and approved the final manuscript.

\section{Funding}

We are planning to submit the results of this study, which was funded by a research grant in 2013 from the Japanese Association of Dialysis Physicians (JADP2013-14), to the Journal of Vascular Access as an original article. To avoid duplicate submissions, the report for the JADP provides an outline of the study.

\section{Availability of data and materials}

The datasets used and/or analyzed during the current study are available from the corresponding author on reasonable request.

\section{Ethics approval and consent to participate}

The authors of this manuscript declare that they have complied with the Principles of Ethical Publishing presented in the Declaration Helsinki and that the study protocol was approved by a local ethics committee. This study was approved by the Ethics Committee of the Juntendo University (approval no. 13-085) and registered with the University Hospital Medical Information Network (UMIN000011965).

\section{Consent for publication}

All patients, or their legally acceptable representatives, provided informed consent prior to entering the study.

\section{Competing interests}

The authors declare that they have no competing interests.

\section{Author details}

'Department of Nephrology, Juntendo University Nerima Hospital, 3-1-10 Takanodai Nerima-ku, Tokyo 177-0033, Japan. ' 2 Department of Nephrology, Juntendo University Faculty of Medicine, Tokyo, Japan. ${ }^{3}$ Medical Corporation Showakai, Tokyo, Japan.

Received: 16 December 2018 Accepted: 24 May 2019

Published online: 16 July 2019

\section{References}

1. Lin CC, Chung MY, Yang WC, Lin SJ, Lee PC. Length polymorphisms of heme oxygenase-1 determine the effect of far-infrared therapy on the function of arteriovenous fistula in hemodialysis patients: a novel physicogenomic study. Nephrol Dial Transplant. 2013;28(5):1284-93.

2. Windus DW. Permanent vascular access: a nephrologist's view. Am J Kidney Dis. 1993;21(5):457-71.

3. Imamura M, Biro S, Kihara $T$, et al. Repeated thermal therapy improves impaired vascular endothelial function in patients with coronary risk factors. J Am Coll Cardiol. 2001;38(4):1083-8.

4. Yu SY, Chiu JH, Yang SD, Hsu YC, Lui WY, Wu CW. Biological effect of farinfrared therapy on increasing skin microcirculation in rats. Photodermatol Photoimmunol Photomed. 2006;22(2):78-86.

5. Lin CC, Chang CF, Lai MY, Chen TW, Lee PC, Yang WC. Far-infrared therapy: a novel treatment to improve access blood flow and unassisted patency of arteriovenous fistula in hemodialysis patients. J Am Soc Nephrol. 2007;18(3): 985-92. 
6. Masuda A, Miyata M, Kihara T, Minagoe S, Tei C. Repeated sauna therapy reduces urinary 8-epi-prostaglandin F(2alpha). Jpn Heart J. 2004;45:297-303.

7. Chen CF, Yang WC, Lin CC. An update of the effect of far infrared therapy on arteriovenous access in end-stage renal disease patients. J Vascs Access. 2016;17(4):293-8

8. Chang CJ, Ko YS, Ko PJ, Hsu LA, Chen CF, Yang CW, Hsu TS, Pang JH. Thrombosed arteriovenous fistula for hemodialysis is characterized by a marked inflammatory activity. Kidney Int. 2005;68(3):1312-9.

9. Napoli C, D'Armiento FP, Mancini FP, Postiglione A, Witztum JL, Palumbo G, Palinski W. Fatty streak formation occurs in human fetal aortas and is greatly enhanced by maternal hypercholesterolemia. Intimal accumulation of low density lipoprotein and its oxidation precede monocyte recruitment into early atherosclerotic lesions. J Clin Invest. 1997;100(11):2680-90.

10. Abdel-Magied N, Ahmed AG, Shedid SM. Near-infrared heat lamp therapeutic effect on paraoxonase 1 and myeloperoxidase as potentia biomarkers of redox state changes induced by $\gamma$-irradiation in albino rats. $J$ Photochem Photobiol. 2018;179:105-12.

\section{Publisher's Note}

Springer Nature remains neutral with regard to jurisdictional claims in published maps and institutional affiliations.

Ready to submit your research? Choose BMC and benefit from:

- fast, convenient online submission

- thorough peer review by experienced researchers in your field

- rapid publication on acceptance

- support for research data, including large and complex data types

- gold Open Access which fosters wider collaboration and increased citations

- maximum visibility for your research: over $100 \mathrm{M}$ website views per year

At BMC, research is always in progress.

Learn more biomedcentral.com/submissions 